This item was submitted to Loughborough's Research Repository by the author.

Items in Figshare are protected by copyright, with all rights reserved, unless otherwise indicated.

\title{
The mechanism of striation formation in plasma display panels
}

PLEASE CITE THE PUBLISHED VERSION

PUBLISHER

(C) American Institute of Physics

LICENCE

CC BY-NC-ND 4.0

REPOSITORY RECORD

Iza, Felipe, S.S. Yang, H.C. Kim, and Jae Koo Lee. 2019. "The Mechanism of Striation Formation in Plasma Display Panels". figshare. https://hdl.handle.net/2134/3217. 
This item was submitted to Loughborough's Institutional Repository by the author and is made available under the following Creative Commons Licence conditions.

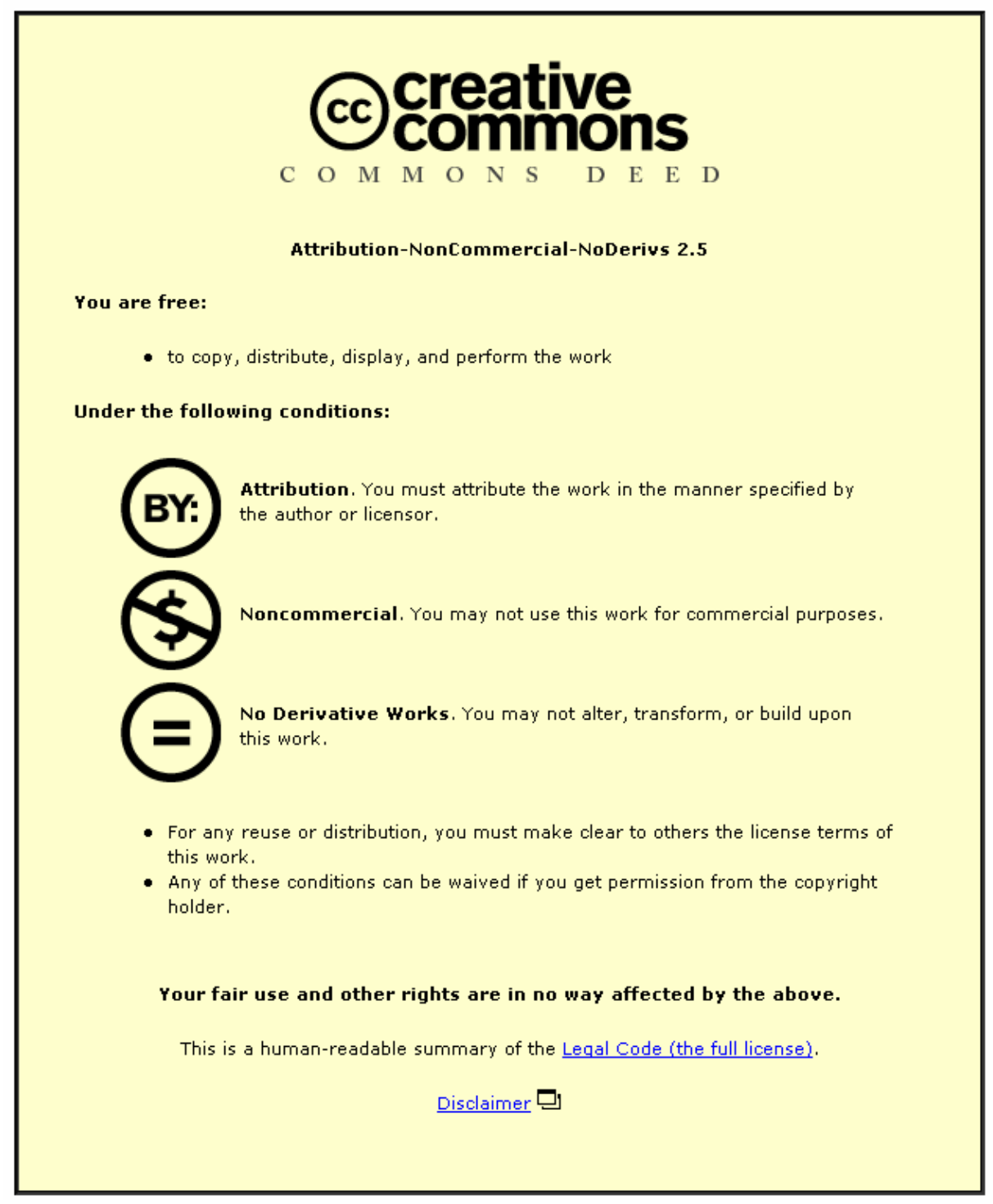

For the full text of this licence, please go to: http://creativecommons.org/licenses/by-nc-nd/2.5/ 


\title{
The mechanism of striation formation in plasma display panels
}

\author{
F. Iza, S. S. Yang, H. C. Kim, and J. K. Lee ${ }^{\text {a) }}$ \\ Electronics and Electrical Engineering Department, Pohang University of Science and Technology, \\ Pohang 790-784, Republic of Korea
}

(Received 19 January 2005; accepted 4 July 2005; published online 18 August 2005)

\begin{abstract}
Despite the high pressure employed in plasma display panels, the energy balance of low-energy electrons is found to be dominated by inelastic collisions, and the resulting nonlocal electron kinetics plays a key role in the striation formation. Surface charge accumulation on the anode dielectric, however, is also needed for striations to form. It is the combined effect of surface charges and nonlocal electron kinetics that results in the striation formation in plasma display panel cells. Two-dimensional fluid simulations, which assume local electron kinetics, and two-dimensional particle-in-cell Monte Carlo collision simulations with a bare conducting anode show that striations do not form if either the nonlocal electron kinetics or the surface charge accumulation is not considered. (C) 2005 American Institute of Physics. [DOI: 10.1063/1.2007851]
\end{abstract}

\section{INTRODUCTION}

The reasons leading to the formation of striations in plasmas are, in general, complex. Ion waves, attachment, and stepwise ionization are known mechanisms that can lead to the formation of striations. ${ }^{1,2}$ Nonlocal electron kinetics has also been successfully presented as a mechanism responsible for the striation formation in positive column discharges. ${ }^{3}$ Phenomena due to nonlocal electron kinetics, however, are typically observed at pressures much lower than those employed in plasma display panels (PDPs). ${ }^{3-6}$

Given the small dimensions of a PDP cell and the short life of the discharge, experimental diagnostics are challenging. For this reason, computer simulation has emerged as an important tool for studying PDP cells. The simulation results presented in this work are obtained using XOOPIC, ${ }^{7}$ a twodimensional self-consistent electromagnetic particle-in-cell Monte Carlo collision (PIC-MCC) simulation program. PICMCC simulations capture the kinetics of the simulated species with very few approximations ${ }^{8}$ and only PIC-MCC simulations have been able to reproduce the striation formation in PDP cells. ${ }^{9-11}$

Although various factors such as gas composition, pressure, and cell geometry are known to affect the number, position, and shape of the striations in PDP cells, the mechanism responsible for their formation, however, continues to be unresolved. Instabilities on the cathode fall ${ }^{12}$ and residual surface charges from previous discharges have been proposed as possible mechanisms for the striation formation in PDP cells. Simulation results, however, have shown that striations form even when no instabilities or oscillations are observed in the cathode region and without preaccumulated surface charges on the dielectrics. ${ }^{11}$ Dielectric surface charges and spatial potential modulations have also been pointed as factors involved in the striation formation. ${ }^{11-13} \mathrm{Up}$ to this day, however, no satisfactory self-consistent explanation has been presented.

In this article, the striation formation mechanism in PDP

${ }^{a)}$ Electronic mail: jkl@postech.ac.kr cells is analyzed and the role of the key factors leading to the striation formation is identified. Although at pressures above a few Torrs nonlocal effects are typically negligible due to a large number of elastic collisions, ${ }^{14}$ nonlocal electron kinetics are found to be partially responsible for the formation of striations in PDP cells even at pressures close to atmospheric pressure. It is the combined effect of surface charges and nonlocal electron kinetics that results in the striation formation.

The rest of this article is arranged as follows: Section II presents a description of the striation formation mechanism indicating the role of surface charges and nonlocal electron kinetics. In Sec. III, the effects of pressure and gas chemistry are considered. In addition, the spatial distribution of the electron temperature is presented. Finally, conclusions are drawn in Sec. IV.

\section{THE MECHANISM OF STRIATION FORMATION: SURFACE CHARGES AND NONLOCAL ELECTRON KINETICS}

Figures 1(a) and 1(d) show a schematic of a coplanar and a matrix-type PDP cell, respectively. The dimensions displayed are the ones used in the simulations and correspond to typical dimensions of actual cells. In this work, we shall refer to ion bunches as striations. Bunches can also be observed in other species such as electrons and excited species. Bunches of different species, however, present slightly different time evolutions. Figures 1(b) and 1(e) show the simulated spatiotemporal evolution of the neon ion density [integrated along the $Y$ direction defined in Figs. 1(a) and $1(d)]$ in a pure neon discharge at 500 Torr. Striations form sequentially following the ignition of the main discharge. They appear along the anode region and become weaker as they form closer to the outer edge of the cell. The lowering of the applied voltage in the discharge region due to the charges accumulated on the dielectric surface causes the eventual extinction of the plasma. Typical PDP discharges last a few microseconds and during this time striations are found to be stationary. The main discharge in a coplanar cell, 
(a)

(b) 775 Ne ion density
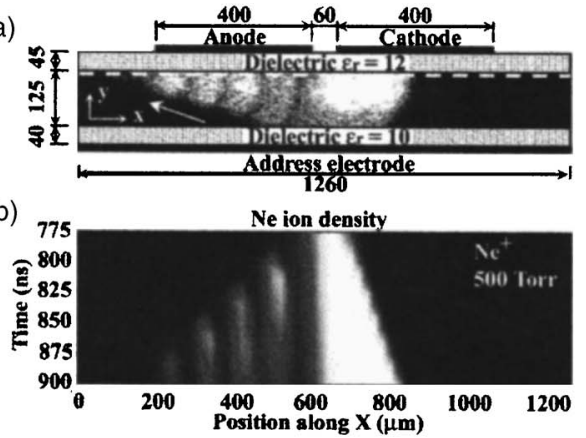

(c)

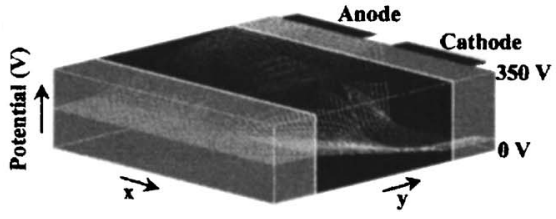

(d)

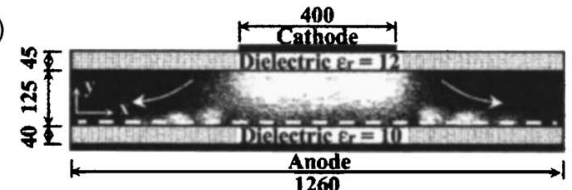

(e)

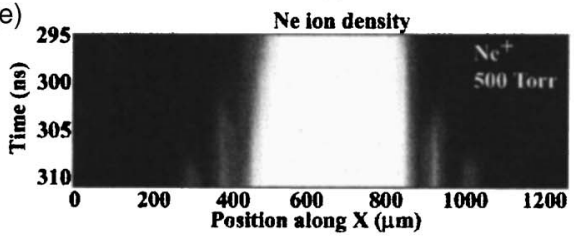

(f)

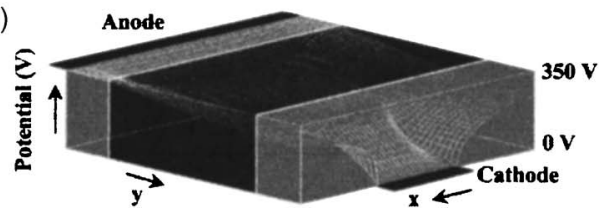

FIG. 1. Striated microdischarge in $[(\mathrm{a})-(\mathrm{c})]$ coplanar and $[(\mathrm{d})-(\mathrm{f})]$ matrix plasma display panel cells operating in $\mathrm{Ne}$ at 500 Torr. Dimensions are given in micrometers, figures are not drawn to scale, and $X$ and $Y$ axes are defined in (a) and (d). The dashed lines in (a) and (d) correspond to planes parallel to the anode dielectric at a distance of $15 \mu \mathrm{m}$. The spatiotemporal evolution of the neon ion density (integrated along $Y$ ) is shown in (b) and (e) and the spatial potential profiles after the striations have formed in (c) and (f). however, shifts towards the cathode region as time progresses [Fig. 1(b)]. These simulation results are in agreement with experimental observations. ${ }^{12,13,15}$ The spatial potential profiles resulting after the striation formation in a coplanar and in a matrix PDP cell are displayed in Figs. 1(c) and 1(f), respectively. Despite the different appearances of the striations in the coplanar and the matrix-type cells, the physics governing the striations is the same in both geom- etries. Figure 2 shows the simulated time evolution of the electron density, the neon ion density, the spatial potential distribution, and equipotential lines during the formation of the first striations in a coplanar PDP cell running in neon at 500 Torr. The evolution of the discharge and the striation formation is described next.

The discharge in a PDP cell is ignited by applying a voltage difference on the order of $\sim 350 \mathrm{~V}$ between the sus-
ELECTRON DENSITY

(a)

TIME $775 n s$

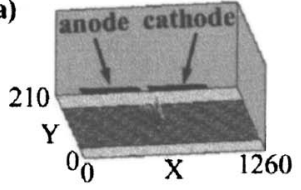

TIME

$790 \mathrm{~ns}$

(b)

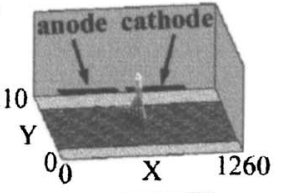

(c)

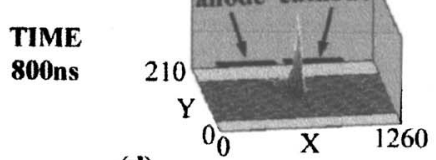

(d)

TIME

810ns

(e)

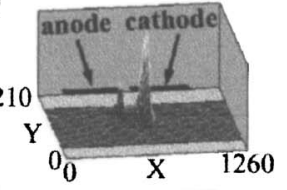

TIME

820ns

TIME

830ns

(f)

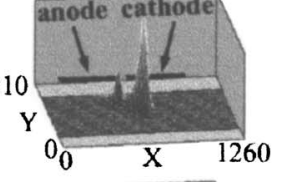

\section{ION DENSITY}

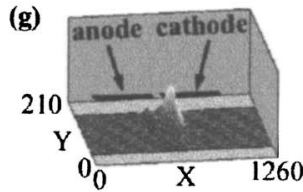

(h)

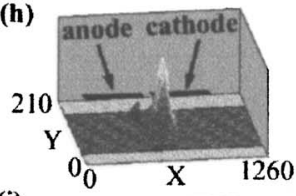

(i)

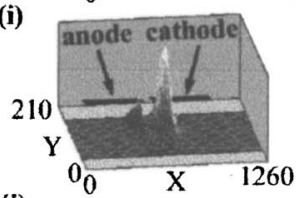

(j)

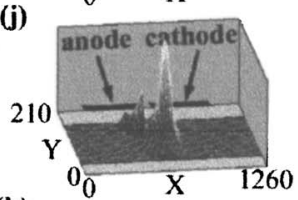

(k)

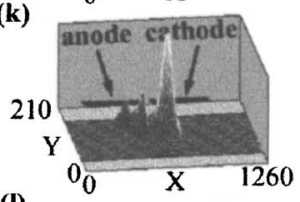

anode cathode (I)

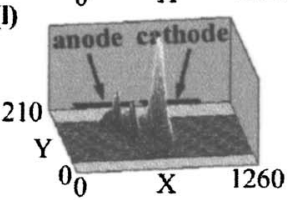

POTENTIAL PROFILE

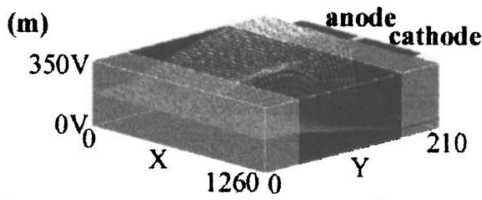

(n)

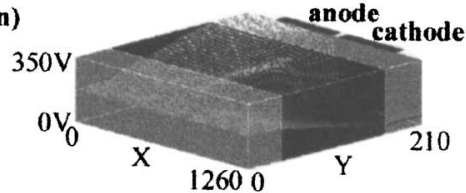

(o)

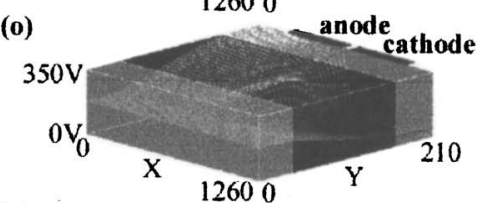

(p)

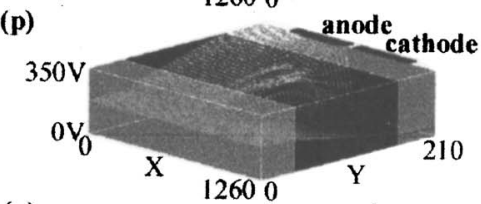

(q)

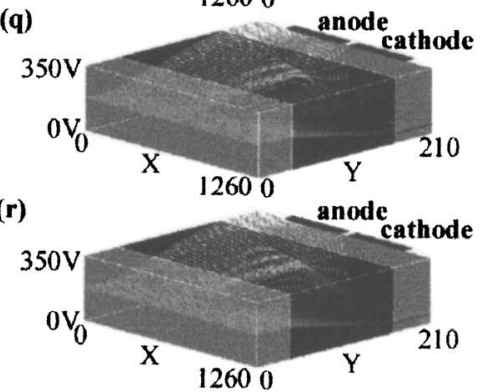

\section{EQUIPOTENTIAL LINES}

(s)

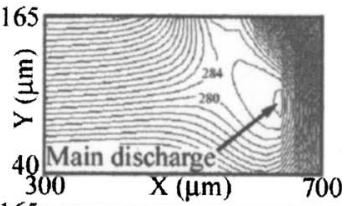

(t)

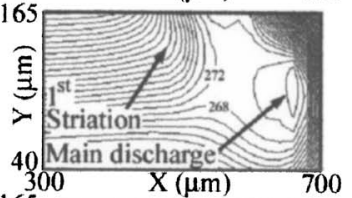

(u)

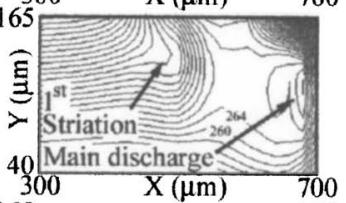

(v)

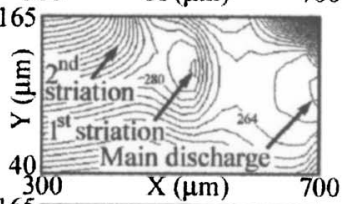

(w)

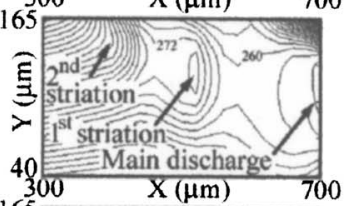

(x)

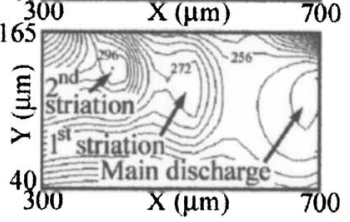

FIG. 2. Time evolution (by rows) of [(a)-(f)] the electron density, $[(\mathrm{g})-(\mathrm{l})]$ the $\mathrm{Ne}^{+}$ion density, $[(\mathrm{m})-(\mathrm{r})]$ the potential profile, and $[(\mathrm{s})-(\mathrm{x})]$ equipotential lines in a coplanar PDP neon discharge at 500 Torr. 
tain electrodes. These electrodes have been labeled as anode and cathode in Figs. 1(a) and 1(d) to indicate the polarity of the applied voltage. The address electrode in the coplanar cell was kept biased to half the applied voltage, i.e., $175 \mathrm{~V}$, in all simulations. The applied voltage between the sustain electrodes causes electrons to drift toward the anode and creates an avalanche that is sustained by the generation of secondary electrons in the cathode region. This avalanche creates the main discharge, which appears on the upper center region of the cell close to the cathode (Fig. 2-time of $775 \mathrm{~ns}$ ). As electrons from the main discharge drift toward the anode, they accumulate on the dielectric layer that covers this electrode. In a coplanar cell [Fig. 1(a)], the charge accumulates in the anode inner edge region, whereas in a matrix cell [Fig. 1(d)] it accumulates in the center anode region. This charge accumulation drives the surface of the dielectric negatively with respect to the plasma potential in the main discharge [Fig. 2(s)]. As a result, low-energy electrons are trapped in the cell and drifted toward outer regions of the anode where surface charge has not yet been accumulated and therefore remain at a higher potential. The extension of the discharge toward the outer edge of the anode due to surface charges is a crucial first step in the striation formation process. In fact, if charge accumulation is eliminated, simulation results show that the discharge does not extend along the anode and that striations do not form. No striations are obtained experimentally either if bare electrodes are employed. ${ }^{12,21}$ Therefore, surface charges are necessary for striations to form in PDPs but on their own do not justify the formation of striations.

The low-energy electrons that are drifted toward the outer region of the anode do not have initially enough energy to ionize the gas. Therefore a low ion density region is created adjacent to the main discharge. As the electrons are accelerated by the electric field, however, they eventually acquire sufficient energy to ionize the gas. At this point, a large number of ionization collisions take place and the first striation forms (Fig. 2-time of 790-800 ns).

In the first instances of the newly formed striation, a significant number of electrons drift toward the anode and accumulate on the dielectric surface in the vicinity of the striation. As a result of this initially high electron loss, the electron density in the newly formed striation is much lower than the ion density [Figs. 2(b), 2(c), 2(h), and 2(i)]. As charge builds up on the dielectric, however, low-energy electrons are confined to the striation region and an increase in the electron density is observed [Figs. 2(d) and 2(v)]. Nevertheless, striations remain ion-rich regions, i.e., regions with net positive charge. As a result, the spatial potential distribution existing prior to the formation of a striation gets modulated by the presence of the striation [Figs. 2(p) and 2(v)]. Since charge accumulates on the dielectric surface primarily in the regions close to a striation, surface charge is not uniformly distributed. ${ }^{11}$ It should be noticed, however, that the uneven charge accumulation is a result of the striation formation in the cell and not its cause.

As with the main discharge, high-energy electrons continue to reach the dielectric in the vicinity of the striation while low-energy ones are trapped in the cell and drifted towards the outer region of the anode [Fig. 2(w)]. A region of low ionization is created next to the first striation due to the initially low energy of the electrons being drifted toward the outer edge of the anode. Once these electrons gain sufficient energy, a new striation forms [Fig. 2(k)]. One can easily see that the process repeats itself leading to the formation of more striations until the outer edge of the anode is reached. The lowering of the applied voltage in the discharge region due to the charges accumulated on the dielectric surface causes the eventual extinction of the discharge.

Prior to the formation of the striations, the electric field (slope of the potential profiles) is uniform in the vicinity of the existing discharge [Figs. 2(m)-2(x)]. Therefore, if electrons were in local equilibrium with the field, uniform ionization would take place and no striations would form, i.e., the discharge would extend along the anode due to surface charge accumulation but would be uniform. It should be noted that the modulation of the potential and the electricfield profiles occurs after the striations have formed. As shown in Fig. 2, the striations are observable in the ion density profile well before the potential profile gets modulated. Therefore, modulations of the spatial potential profile are a consequence of the striation formation and not its cause as has been suggested. ${ }^{13}$

In high-pressure discharges, the electron mean energy (temperature) is normally found to depend on the reduced electric field $(E / n) .{ }^{1}$ In PDP cells and despite the high pressure of operation ( $\sim 500$ Torr), electrons gain energy as they drift towards the outer edge of the anode with negligible energy loss in collisional processes between striations (this statement is justified below). As a consequence, the energy of the electrons is determined by the potential distribution instead of the reduced electric field, i.e., electrons are not in local equilibrium with the electric field. This is the underlying physical reason for the striation formation.

It can be observed in the spatial potential distribution along a plane parallel to the dielectric surface $15 \mu \mathrm{m}$ inside the cell (Fig. 3) that the potential difference between the location of one striation and the future location of the next striation is slightly larger than the ionization potential of the gas $(22 \mathrm{eV}$ for $\mathrm{Ne})$. This indicates that the energy gained by the electrons as they are accelerated by the electric field between these two points is effectively retained despite collisions and that electrons are not in equilibrium with the field.

For a typical pressure in a PDP cell of $\sim 500$ Torr and a maximum collision cross section for $\mathrm{Xe}-\mathrm{Ne}$ mixtures of 3 $\times 10^{-19} \mathrm{~m}^{2},{ }^{16}$ the minimum electron mean-free path is $\sim 0.2 \mu \mathrm{m}$. Therefore, electrons undergo only a few hundred collisions as they drift between striations [striation separation is $\sim 100 \mu \mathrm{m}$-Figs. 3(a) and 3(b)]. Since the electrons responsible for the striation formation are low-energy electrons (high-energy electrons are less in number and are capable to escape and accumulate on the dielectric in the vicinity of existing striations), these collisions are elastic.

It is well known that due to the large mass difference between electrons $\left(m_{e}\right)$ and neutrals $(M)$, the energy transfer from an electron to a neutral in an elastic collision is very inefficient. ${ }^{17}$ Nevertheless, elastic collisions can limit the mean energy an electron can acquire in a given electric field. 
(a)

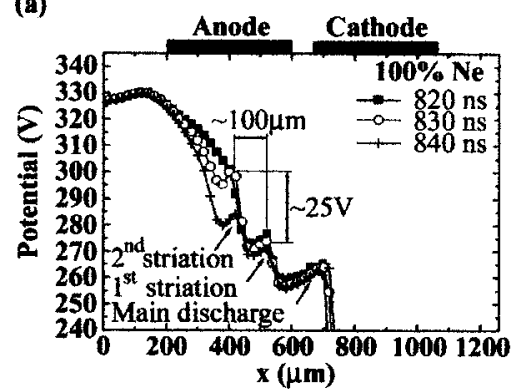

(b)

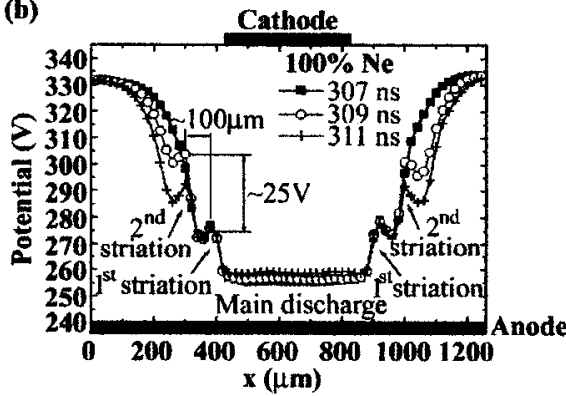

(c)

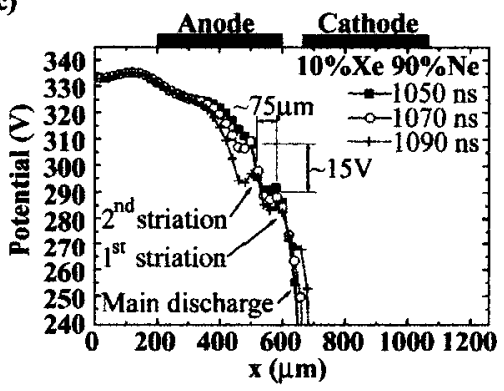

FIG. 3. Time evolution of the spatial potential distribution in the anode region for (a) $100 \%$ Ne discharge in a coplanar cell, (b) $100 \%$ Ne discharge in a matrix cell, and (c) $10 \% \mathrm{Xe}-90 \% \mathrm{Ne}$ discharge in a coplanar cell. Potentials lines are plotted for a plane parallel to the anode dielectric at a distance of $15 \mu \mathrm{m}$ [dashed lines in Figs. 1(a) and 1(d)].

It can be shown that an electron starting at rest undergoes $\sim M / m_{e}\left(\sim 10^{4}\right)$ collisions before reaching $60 \%$ of its maximum (equilibrium) mean energy and that the energy lost in a few hundred collisions represents only a few percent of the energy the electrons would gain in a collisionless case. Therefore, and although at high pressures the electron mean energy is typically determined by the reduced electric field $(E / n){ }^{1}$ the electron mean energy in PDP cells is determined by the potential distribution, i.e., electrons are not in local equilibrium with the electric field. This nonlocality of lowenergy electrons also explains why fluid simulations, which are based on the local-field approximation, have failed to reproduce the striation phenomenon in PDP cells. ${ }^{9-11}$

\section{DISCUSSION}

The mechanism presented in Sec. II provides a better understanding on the influence of various physical quantities on the striation formation. Gas composition and pressure are known to affect the number and shape of the striations and their influence has been examined by simulating Xe-Ne mixtures and discharges at 100-500 Torr. The following conclusions are drawn.

The gas composition affects the striation separation by setting the distance electrons need to travel in a given field to acquire the ionization energy [compare Figs. 3(a) and 3(c) for the cases of $100 \% \mathrm{Ne}$ and $10 \% \mathrm{Xe}-90 \% \mathrm{Ne}$. As a consequence of the markedly lower ionization potential of $\mathrm{Xe}$ $(12 \mathrm{eV})$, striations form closer together in the mixture discharge [compare Figs. 1(b) and 4(a)]. Although xenon partial concentration is smaller than that of neon, xenon ions are more abundant than neon ions due to the lower ionization potential of xenon. As a result, striations in the Ne ion density are less marked than those in Xe and are not presented. This has also been observed experimentally. ${ }^{13}$

In agreement with experimental observations, ${ }^{12,13}$ the number of striations in the simulations increases with pressure. The increase in the number of striations is due to a reduction on the striation width (along $X$ direction in Fig. 1) and not to a reduction of the separation between striations. The separation between the striations is independent of pressure and it is determined by the spatial voltage distribution and the ionization potential of the gas. As pressure increases, however, the ionization mean-free path decreases $\left(\lambda_{\mathrm{iz}}\right.$ $\sim 1 / P)$. Therefore, once electrons reach the ionization en- ergy, they ionize the gas within a shorter distance at higher pressure. As a result, narrower striations are observed at high pressure. For the geometry depicted in Fig. 1(a), the number of striations in a $10 \% \mathrm{Xe}-90 \% \mathrm{Ne}$ discharge increases by one (from 4 to 5) as pressure is increased from 200 to 500 Torr (Fig. 4).

For the geometry and conditions simulated in this work, the time span between the appearance of subsequent striations is on the order of $\sim 25 \mathrm{~ns}$ in the coplanar cell and $\sim 5 \mathrm{~ns}$ in the matrix cell [Figs. 1(b), 1(e), and 4]. As explained in Sec. II, the propagation of the discharge toward the outer anode region is caused by the charging of the dielectric, and therefore striation appearance will depend on the density of the discharge and the three-dimensional geometry of the cell. Time intervals observed in experiments range from a few up to a few hundred nanoseconds. ${ }^{12,13,18}$ It should be noted, however, that experimental observations are normally carried out in multipulse operation whereas simulation results are typically presented for a single pulse. For a comparison between simulation results and experimental data, one should consider the residual surface charge from previous pulses as this will affect the ignition and propagation speed of the discharge.

The modulation of the potential distribution once stria-
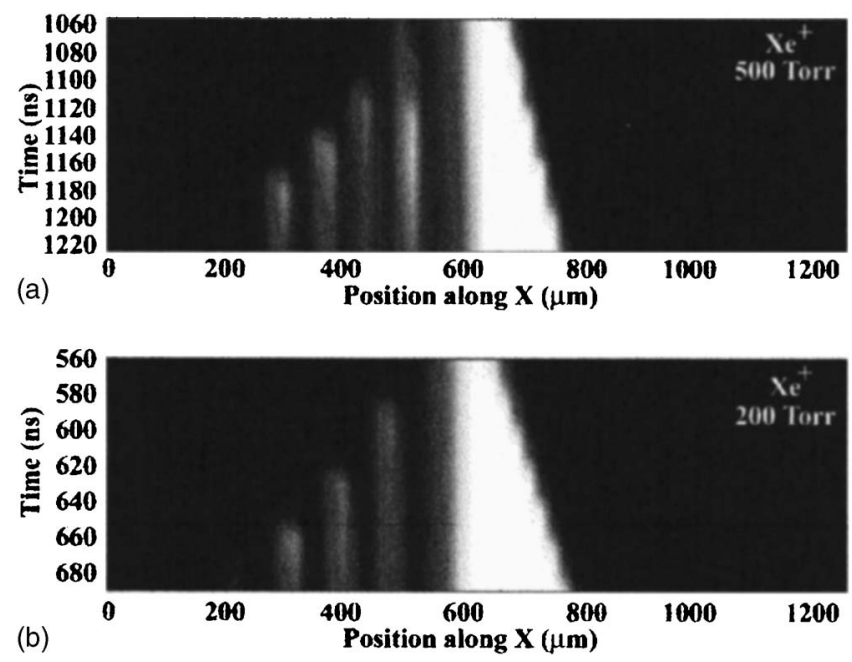

FIG. 4. Spatiotemporal evolution of $\mathrm{Xe}^{+}$ion density (integrated along $Y$ ) in a $10 \% \mathrm{Xe}-90 \% \mathrm{Ne}$ discharge at (a) 500 Torr and (b) 200 Torr. Cell geometry and $Y$ direction shown in Fig. 1(a). 


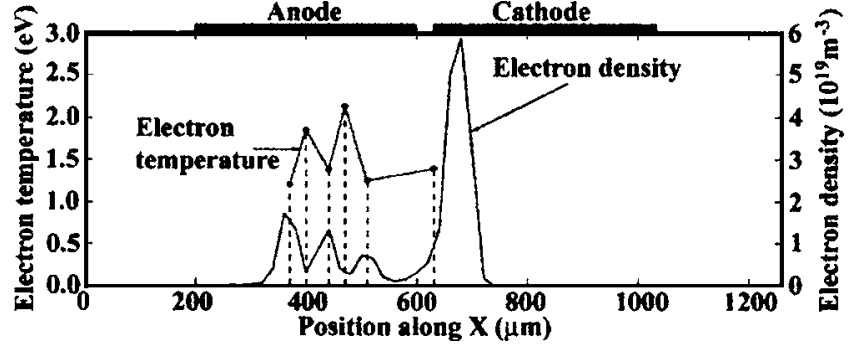

FIG. 5. Electron density and temperature for a striated 10\% Xe-90\% Ne discharge at 500 Torr in a coplanar PDP cell. Data shown for a plane parallel to the dielectric $15 \mu \mathrm{m}$ inside the discharge [dashed line in Fig. 1(1)].

tions have formed results in a higher electric field in the interstriation regions than in the striations themselves (slope of the potential profiles in Figs. 2 and 3). Although electrons do not reach equilibrium with the electric field, the few hundred collisions they undergo as they are accelerated in the interstriation regions randomize their increased velocity and thereby increase the electron temperature. As a result, the electron temperature in between striations is higher than in the striations themselves. Figure 5 shows the electrondensity profile and the electron temperature along a plane parallel to the dielectric in a $10 \% \mathrm{Xe}-90 \% \mathrm{Ne}$ discharge at 500 Torr. Both profiles are out-of-phase as recently observed in laser Thompson scattering measurements. ${ }^{19}$ Since both electron temperature and plasma density determine the UV radiation from excited species, ${ }^{20}$ these modulations along striations affect the luminous efficiency of the cell.

\section{CONCLUSION}

In conclusion, despite the high pressure employed in PDP cells ( $\sim 500$ Torr), low-energy electrons are found not to be in local equilibrium with the electric field. This is a consequence of the large electric fields generated in these microdischarges. Although nonlocal kinetics on its own has explained the formation of striations in low-pressure positive-column discharges, in PDP cells striations do not form without the combined effect of surface charges. Surface charges reduce the electron loss to the anode dielectric by generating a trapping potential well for low-energy electrons. This allows the extension of the discharge along the anode, a necessary first step in the striation formation. On their own, however, surface charges do not explain the striation formation either. It is therefore the combined effect of nonlocal kinetics and surface charges that result in the striation formation in PDP cells. If one of these two factors is missing, simulations show that striations do not form.

The presented mechanism of striation formation satisfactorily agrees with previously reported observations such as the uneven charge accumulation on the dielectric in the anode region, the successive modulations of the spatial poten- tial, and the pressure and gas composition dependence. The mechanism is equally applicable to the coplanar and the matrix-type PDP cells, and it can be extended to other geometries/applications. Based on the understanding of the mechanism leading to the extension of the discharge along the anode in a PDP cell, one can envision covering an electrode with a dielectric as a technique for extending microdischarges along capillaries or hollow anodes as those employed in. ${ }^{21}$ As in the case of PDP cells and despite the high pressure of operation, nonlocal kinetics of low-energy electrons may also play a role in the striation formation in other microscale discharges where electric-field strengths even larger than those found in PDP cells are employed. ${ }^{22}$

\section{ACKNOWLEDGMENTS}

This work was supported by LG Electronics and the Korean Ministry of Science and Technology under the Teralevel Nano Devices Project-21C Frontier R\&D Program.

${ }^{1}$ Y. P. Raizer, Gas Discharge Physics (Springer, Berlin, New York, 1991). ${ }^{2} \mathrm{M}$. M. Turner and D. Vender, Abstracts for the IEEE International Conference on Plasma Science, June 2000, IEEE International Conference on Plasma Science, New Orleans, LA, 4-7 June 2000 (IEEE, New York, 2000), p. 163.

${ }^{3}$ V. I. Kolobov and V. A. Godyak, IEEE Trans. Plasma Sci. 23, 503 (1995).

${ }^{4}$ H. C. Kim and J. K. Lee, Phys. Rev. Lett. 93, 085003 (2004).

${ }^{5}$ H. C. Kim, G. Y. Park, and J. K. Lee, Appl. Phys. Lett. (submitted).

${ }^{6}$ Y. B. Golubovskii, V. A. Maiorov, I. A. Porokhova, and J. Behnke, J. Phys. D 32, 1391 (1999).

${ }^{7}$ J. P. Verboncoeur, A. B. Langdon, and N. T. Gladd, Comput. Phys. Commun. 87, 199 (1995).

${ }^{8}$ C. K. Birdsall and A. B. Langdon, Plasma Physics Via Computer Simulation (IOP, Bristol, UK, 1991).

${ }^{9}$ S. Rauf and M. J. Kushner, J. Appl. Phys. 85, 3460 (1999); 85, 3470 (1999).

${ }^{10}$ C. Punset, J. P. Boeuf, and L. C. Pitchford, J. Appl. Phys. 83, 1884 (1998). ${ }^{11}$ C. H. Shon and J. K. Lee, Phys. Plasmas 8, 1070 (2001).

${ }^{12}$ G. Cho, E. H. Choi, Y. G. Kim, D. I. Kim, H. S. Uhm, Y. D. Joo, J. G. Han, and M. C. Kim, J. Appl. Phys. 87, 4113 (2000).

${ }^{13}$ T. Yoshioka, A. Okigawa, L. Tessier, and K. Toki, Proceedings of the 6 th International Display Workshop, Sendai, Japan, 1-3 December 1999 (Society for Information Display, San Jose, CA, 1999), p. 603.

${ }^{14}$ Y. B. Golubovskii, V. A. Maiorov, I. A. Porokhova, and J. Behnke, J. Phys. D 32, 1391 (1999).

${ }^{15}$ Th. Callegari, R. Ganter, and J. P. Boeuf, J. Appl. Phys. 88, 3905 (2000).

${ }^{16}$ Kinema Software, http://www.siglo-kinema.com/bolsig.htm

${ }^{17}$ M. A. Lieberman and A. J. Lichtenberg, Principle of Plasma Discharges and Materials Processing (Wiley, New York, 1994).

${ }^{18}$ R. Ganter, J. Ouyang, Th. Callegari, and J. P. Boeuf, J. Appl. Phys. 91, 992 (2002).

${ }^{19}$ S. Hassaballa, Y. K. Kim, Y. Yamagata, K. Uchino, H. Hatanaka, Y. M. Kim, S. E. Lee, S. H. Son, and S. H. Jang, Proceedings of the 11th International Display Workshop, Niigata, Japan, 8-10 December 2004 (Society for Information Display, San Jose, CA, 1999), p. 863.

${ }^{20}$ K. Tachibana, S. Feng, and T. Sakai, J. Appl. Phys. 88, 4967 (2000).

${ }^{21}$ R. M. Sankaran, K. P. Giapis, D. Holunga, and R. C. Flagan, AVS 51st International Symposium and Exhibition, Anaheim, CA, 14-19 November 2004 (AVS The Science and Technology Society, New York, 2004), p. 179.

${ }^{22}$ F. Iza and J. Hopwood, IEEE Trans. Plasma Sci. 33, 306 (2005). 